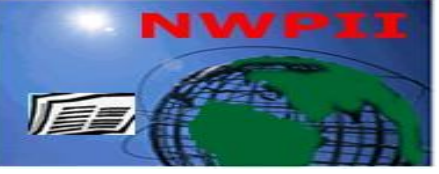

American Journal of Biomedical Sciences

ISSN: 1937-9080

nwpii.com/ajbms

\title{
Induction of Apoptosis and Autophagy in Human Pancreatic Cancer Cells by a Novel Synthetic C-1 Analogue of 7-deoxypancratistatin
}

\section{Dennis Ma ${ }^{1}$, Phillip Tremblay ${ }^{1}$, Kevinjeet Mahngar ${ }^{1}$, Pardis Akbari-Asl ${ }^{1}$, Jonathan Collins ${ }^{2}$, Tomas Hudlicky ${ }^{2}$, and Siyaram Pandey ${ }^{*}$}

\author{
${ }^{1}$ Department of Chemistry and Biochemistry, University of Windsor, Windsor, Ontario, Canada \\ ${ }^{2}$ Chemistry Department and Centre for Biotechnology, Brock University, St. Catharines, Ontario, Canada \\ *Corresponding Author: \\ Dr. Siyaram Pandey, Professor \\ Department of Chemistry and Biochemistry \\ University of Windsor \\ 401 Sunset Avenue \\ Windsor, Ontario N9B 3P4 \\ Canada
}

Phone: +519-253-3000, ext. 3701

Email: spandey@uwindsor.ca

Received: 13 June 2011; | Revised: 25 August 2011; | Accepted: 5 September 2011

\begin{abstract}
Pancreatic cancer is amongst the deadliest cancers in the world. It is associated with poor prognosis, is notorious for developing chemoresistance, and very few approved chemotherapeutics are available to treat this disease. The natural compound pancratistatin (PST) has shown to effectively induce cytotoxicity selectively in numerous cancer cell types. However, it is present in only minute quantities in its natural source and many complications have burdened its chemical synthesis. We have overcome these bottlenecks by synthesizing a C-1 acetoxymethyl analogue of 7-deoxypancratistatin, JC-TH-acetate-4 (JCTH-4), which we have shown to have similar selective anti-cancer activity to that of PST. In this report, we show JCTH-4 to be a potent chemotherapeutic against pancreatic cancer cells (BxPC-3, PANC-1). It induced apoptosis selectively in BxPC-3 and PANC-1 cells by targeting the mitochondria; it dissipated mitochondrial membrane potential, caused release of apoptogenic factors, and in isolated mitochondria, increased the generation of reactive oxygen species. Furthermore, JCTH-4 selectively induced autophagy in pancreatic cancer cells while normal human fetal fibroblasts were markedly less sensitive to JCTH-4 insult. Altogether, this study outlines JCTH-4 as a potentially safe and effective chemotherapeutic agent in treating notoriously chemoresistant pancreatic cancer.
\end{abstract}

Keywords: Pancreatic cancer, apoptosis, autophagy. 


\section{Introduction}

Type I programmed cell death or apoptosis induction can arise extrinsically, as a result of death receptor ligation at the plasma membrane or intrinsically, as a response to internal cellular stress [1]. Such internal stress, including DNA damage, triggers upregulation of proapoptotic proteins which cause permeabilization of the mitochondrial membrane, dissipation of mitochondrial membrane potential (MMP), and release of apoptogenic factors [2,3]. Once released from the mitochondria, these factors can execute apoptosis directly or indirectly via activation of caspases, a family of cysteine proteases [2,3].

Alternatively, type II programmed cell death, or autophagic cell death, results from the extensive activation of autophagy, a process which normally functions as a pro-survival stress response that allows cells to persist under unfavourable conditions [4]. Autophagy can be triggered by various forms of cellular stress such as oxidative stress, dysfunctional organelles, deficiencies in growth factors and nutrients, hypoxia, and pathogens [5]. Upon autophagic induction a membrane sac, a phagophore, engulfs portions of cytoplasm containing proteins and organelles to be degraded [6]. This gives rise to double-membrane vesicles termed autophagosomes which subsequently fuse with lysosomes to yield autolysosomes; previously engulfed contents are then degraded via lysosomal hydrolases [6]. Avoidance of programmed cell death has been implicated in cancer etiology and restoration or selective induction of these pathways may prove to be advantageous strategies in cancer therapy $[7,8]$.

Pancreatic cancer is notoriously one of the most deadly cancers worldwide [9,10]. Most patients are diagnosed at advanced stages of this disease and consequently few live longer than 6 months post diagnosis [11,12]. The standard therapy for pancreatic cancer has been gemcitabine, increasing quality of life and survival time [13]. However, the largest obstacles in combating this malignancy are inherent and developed resistance against chemotherapy $[14,15]$. Currently, there are only a few other approved chemotherapeutic agents available for treating pancreatic cancer, and thus, it would be invaluable to find and develop novel compounds with greater efficacy and specificity towards these malignancies.

In preceding studies, we have reported the Amaryllidaceae alkaloid pancratistatin (PST) to induce apoptosis selectively in a variety of cancer cell types at low doses and decrease the volume of human tumors in vivo; PST is well tolerated in mice and non-cancerous cells are drastically less sensitive to this compound [16-22]. Nevertheless, the availability of this compound has impeded its preclinical and clinical work; PST is present in only minuscule quantities in the Hymenocallis littoralis plant and many difficulties have been encountered with its chemical synthesis. To overcome these issues, we have produced 7deoxypancratistatin derivatives via chemoenzymatic synthesis from bromobenzene and have screened them for similar anti-cancer activity; one C- 1 acetoxymethyl analogue, JC-THacetate-4 (JCTH-4), was identified to have comparable efficacy and specificity to PST in several cancer cell types [23]. Herein, we report JCTH-4 to be an effective inducer of apoptosis and autophagy selectively in pancreatic cancer cells. Such activity in pancreatic cancer may be attributed to mitochondrial targeting by JCTH-4; this compound caused the release of apoptogenic factors from the mitochondria, dissipated mitochondrial membrane potential, and in isolated mitochondria, increased the production of reactive oxygen species (ROS). Such cytotoxicity was not observed in normal human fetal fibroblasts (NFF). Thus, these results present JCTH-4 as a potential selective and potent chemotherapeutic against pancreatic cancer.

\section{Materials and Methods}

\subsection{Cell Culture}

A pancreatic adenocarcinoma cell line, BxPC3 (American Type Culture Collection, Cat. No. CRL-1687, Manassas, VA, USA), was cultured in RPMI-1640 medium (Sigma-Aldrich Canada, Mississauga, ON, Canada) supplemented with $10 \%(\mathrm{v} / \mathrm{v})$ fetal bovine serum (FBS) standard (Thermo Scientific, Waltham, MA, USA) and 10 
$\mathrm{mg} / \mathrm{mL}$ gentamicin (Gibco BRL, VWR, Mississauga, ON, Canada). A pancreatic epithelioid carcinoma cell line, PANC-1 (American Type Culture Collection, Cat. No. CRL-1469, Manassas, VA, USA), was cultured in Dulbecco's Modified Eagle's Medium (SigmaAldrich Canada, Mississauga, ON, Canada) supplemented with $10 \%$ (v/v) FBS standard (Thermo Scientific, Waltham, MA, USA) and 10 $\mathrm{mg} / \mathrm{mL}$ gentamicin (Gibco BRL, VWR, Mississauga, ON, Canada). Apparently normal human fetal fibroblast (NFF) cells (Coriell Institute for Medical Research, Cat. No. AG04431B, Camden, NJ, USA) were grown in Dulbecco's Modified Eagle's Medium, High Glucose (Thermo Scientific, Waltham, MA, USA) supplemented with $15 \% \quad(\mathrm{v} / \mathrm{v})$ FBS standard (Thermo Scientific, Waltham, MA, USA) and 10 $\mathrm{mg} / \mathrm{mL}$ gentamicin (Gibco BRL, VWR, Mississauga, ON, Canada). All cells were maintained at $37^{\circ} \mathrm{C}$ and $5 \% \mathrm{CO}_{2}$.

\subsection{Cell Treatment}

In this study, cells were cultured to 60-70\% confluence and subsequently treated with tamoxifen (TAM) citrate salt (Sigma-Aldrich Canada, Cat. No. T9262, Mississauga, ON, Canada), the broad spectrum caspase inhibitor ZVAD-FMK (EMD Chemicals, Gibbstown, NJ, USA), and JC-TH-acetate-4 (JCTH-4) at the indicated concentrations and durations. As previously described, JCTH-4 was created by chemoenzymatic synthesis from bromobenzene [23]. Compounds were dissolved in dimethylsulfoxide $\left(\mathrm{Me}_{2} \mathrm{SO}\right)$.

\subsection{Nuclear Staining}

Following treatment and incubation with the aforementioned drugs, cells were incubated with $10 \mu \mathrm{M}$ Hoechst 33342 dye (Molecular Probes, Eugene, OR, USA) for 5 minutes to visualize the nuclei. Images were aquired at 400x magnification on a Leica DM IRB inverted fluorescence microscope (Wetzlar, Germany).

\subsection{Annexin V Binding Assay}

The Annexin V binding assay was carried out to validate apoptosis. After drug treatment, cells were washed with phosphate buffer saline (PBS) twice, resuspended in Annexin $\mathrm{V}$ binding buffer (10 mM HEPES, $10 \mathrm{mM} \mathrm{NaOH}, 140 \mathrm{mM} \mathrm{NaCl}, 1$ $\mathrm{mM} \mathrm{CaCl} 2, \mathrm{pH} 7.6$ ), and incubated with Annexin V AlexaFluor-488 (1:50) (Sigma-Aldrich Canada, Mississauga, ON, Canada) for 15 minutes. Fluorescent micrographs were acquired at 400x magnification on a Leica DM IRB inverted fluorescence microscope (Wetzlar, Germany). WST-1 Assay for Cell Viability

The WST-1 based colorimetric assay was carried out as per the manufacturer's protocol (Roche Applied Science, Indianapolis, IN, USA) to quantify cell viability as a function of active cell metabolism. Clear bottom 96-well tissue culture plates were seeded with approximately 4.0 $\mathrm{x} 10^{3}$ BxPC-3 cells/well, 6.0 x $10^{3}$ PANC-1 cells/well, or $5.0 \times 10^{3} \mathrm{NFF}$ cells/well and subsequently, cells were treated with JCTH-4 and Z-VAD-FMK at the indicated doses and durations. After treatment, the WST-1 reagent, which is converted to formazan by cellular enzymes, was administered into each well and incubated for 4 hours at $37^{\circ} \mathrm{C}$. Absorbance readings were taken at $450 \mathrm{~nm}$ on a Wallac Victor ${ }^{3 \mathrm{TM}} 1420$ Multilabel Counter (PerkinElmer, Woodbridge, ON, Canada) to quantify the formazan product. Absorbance readings were expressed as percentages of the solvent control groups.

\subsection{Tetramethylrhodamine Methyl Ester (TMRM) Staining}

To detect MMP, tetramethylrhodamine methyl ester (TMRM) (Gibco BRL, VWR, Mississauga, ON, Canada) was used. Cells were grown on coverslips and treated with JCTH-4 at the indicated concentrations and for the indicated durations. After treatment, cells were incubated with $200 \mathrm{nM}$ TMRM for 45 minutes at $37^{\circ} \mathrm{C}$. Fluorescent micrographs were taken at 400x magnification on a Leica DM IRB inverted fluorescence microscope (Wetzlar, Germany).

\subsection{Mitochondrial Isolation}

Mitochondria were isolated from untreated BxPC-3 cells. BxPC-3 cells were washed two times in cold $\mathrm{PBS}$, resuspended in hypotonic buffer (1 mM EDTA, $5 \mathrm{mM}$ Tris- $\mathrm{HCl}, 210 \mathrm{mM}$ mannitol, $70 \mathrm{mM}$ sucrose, $10 \mu \mathrm{M}$ Leu-pep, $10 \mu \mathrm{M}$ Pep-A, and $100 \mu \mathrm{M}$ PMSF), homogenized, and 
centrifuged at $600 \times \mathrm{g}$ for 5 minutes at $4^{\circ} \mathrm{C}$. The supernatant was centrifuged at $15,000 \mathrm{x} \mathrm{g}$ for 15 minutes at $4^{\circ} \mathrm{C}$. The resulting cytosolic supernatant was discarded and the mitochondrial pellet was resuspended in cold reaction buffer $(2.5$ $\mathrm{mM}$ malate, $10 \mathrm{mM}$ succinate, $10 \mu \mathrm{M}$ Leu-pep, 10 $\mu \mathrm{M}$ Pep-A, and $100 \mu \mathrm{M}$ PMSF in PBS).

\subsection{Amplex Red Assay}

ROS production was measured with Amplex Red (Molecular Probes, Eugene, OR, USA). Isolated mitochondria suspended in cold reaction buffer were loaded into wells of an opaque 96well plate $(20 \mu \mathrm{g}$ of protein/well) with the indicated concentrations of drugs. Paraquat (PQ) (Sigma-Aldrich Canada, Mississauga, ON, Canada) was used as a positive control at $250 \mu \mathrm{M}$. Amplex Red reagent was added to each well at a final concentration of $50 \mu \mathrm{M}$ and horseradish peroxidase (HRP) (Sigma-Aldrich Canada, Mississauga, ON, Canada) was added in the ratio of $6 \mathrm{U} / 200 \mu \mathrm{L}$. Fluorescence readings were taken after 2 hours of incubation at Ex. $560 \mathrm{~nm}$ and Em. $590 \mathrm{~nm}$ on a spectrofluorometer (SpectraMax Gemini XS, Molecular Devices, Sunnyvale, CA, USA). Fluorescence readings were expressed as relative fluorescence units (RFU).

\subsection{Cellular Lysate Preparation}

After 72 hours of treatment with the indicated concentrations of JCTH-4 and TAM, cells were manually homogenized in cold hypotonic buffer $(10 \mathrm{mM}$ Tris $\mathrm{HCl}$ at $\mathrm{pH} 7.2,5 \mathrm{mM} \mathrm{KCl}, 1 \mathrm{mM}$ $\mathrm{MgCl}_{2}, 1 \mathrm{mM}$ EGTA, $1 \%$ Triton-X-100; $10 \mu \mathrm{M}$ Leu-pep, $10 \mu \mathrm{M}$ Pep-A, and $100 \mu \mathrm{M}$ PMSF). Cell lysates were stored at $-20^{\circ} \mathrm{C}$ until use.

\subsection{Western Blot Analyses}

Protein samples were subjected to SDSPAGE and transferred to a nitrocellulose membrane. Membranes were blocked with a $5 \%$ w/v milk TBST (Tris-Buffered Saline with Tween-20) solution for 1 hour and probed overnight at $4^{\circ} \mathrm{C}$ with either: an anti-LC3 antibody raised in rabbits (1:500) (Novus Biologicals, Cat. No. NB100-2220, Littleton, CO, USA), an anti- $\beta$-Actin antibody raised in mice (1:1000) (Santa Cruz Biotechnology, Inc., Cat. No. sc-81178, Paso Robles, CA, USA), an anti- cytochrome $\mathrm{C}$ (Cyto $\mathrm{C}$ ) antibody raised in mice (1:1000) (Abcam, Cat. No. ab13575, Cambridge, MA, USA), an anti-apoptosis inducing factor (AIF) antibody raised in rabbits (1:1000) (Abcam, Cat. No. ab1998, Cambridge, MA, USA), an antiendonuclease $G$ (EndoG) antibody raised in rabbits (1:1000) (Abcam, Cat. No. ab9647, Cambridge, MA, USA), or an anti-succinate dehydrogenase subunit A (SDHA) antibody raised in mice (1:1000) (Santa Cruz Biotechnology, Cat. No. sc-59687, Paso Robles, CA, USA). Membranes were subjected to one 15 minute and two 5 minute washes in TBST and were incubated with an anti-mouse (1:2000) (Abcam, Cat. No. ab6278, Cambridge, MA, USA) or an anti-rabbit (1:2000) (Abcam, Cat. No. ab6802, Cambridge, MA, USA) horseradish peroxidase-conjugated secondary antibody for 1 hour at $25^{\circ} \mathrm{C}$. Membranes were washed three times for 5 minutes in TBST. Enhanced chemiluminescence reagent (Sigma-Aldrich Canada, CPS160, Mississauga, ON, Canada) was used to visualize the bands. ImageJ software was used to perform densitometry analyses.

\subsection{Monodansylcadaverine (MDC) Staining}

Monodansylcadaverine (MDC) (SigmaAldrich Canada, Mississauga, ON, Canada) was used to visualize autophagic vacuoles. Cells were grown on coverslips and treated with the indicated concentrations of drugs and durations. Subsequent to drug treatment, cells were incubated with $0.1 \mathrm{mM}$ MDC for 15 minutes. Micrographs were acquired at 400x magnification on a Leica DM IRB inverted fluorescence microscope (Wetzlar, Germany).

\subsection{Propidium Iodide (PI) Staining}

Cell lysis was observed using Propidium iodide (PI) dye (Sigma-Aldrich Canada, Mississauga, ON, Canada). For 10 minutes, cells were incubated with PI at a concentration of 1 $\mu \mathrm{g} / \mathrm{mL}$. Subsequently, micrographs were acquired with a Leica DM IRB inverted fluorescence microscope (Wetzlar, Germany) at 400x magnification. 

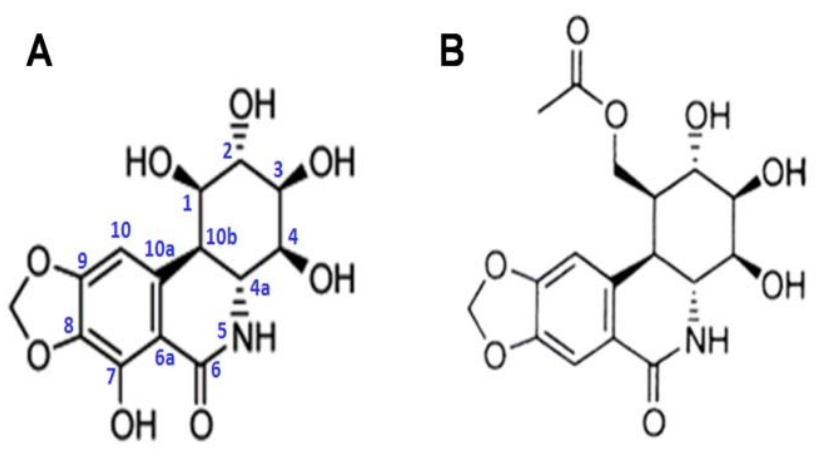

Pancratistatin

(PST)
JC-TH-acetate-4

(JCTH-4)

Figure 1: Comparison of Chemical Structures. The structures of (A) Pancratistatin (PST) and (B) JC-THacetate-4 (JCTH-4).

\section{Results}

\subsection{JCTH-4 Selectively Induces Cytotoxicity in Pancreatic Carcinoma Cells in a Time \& Dose- Dependent Manner}

To circumvent the issues surrounding the low availability of PST (Figure 1A), a potent and selective inducer of cytotoxicity in numerous cancer cell types, synthetic analogues of PST were synthesized and evaluated for similar activity in BxPC-3 and PANC-1 pancreatic cancer cell lines. Using the WST-1 colorimetric assay for cell viability, it was found that JCTH-4 (Figure 1B), a $\mathrm{C}-1$ acetoxymethyl analogue of 7deoxypancratistatin, was able to effectively induce cytotoxicity in BxPC-3 and PANC-1 cells in a time and dose dependent manner (Figure 2A \& B). After 96 hours, JCTH-4 had an approximate halfmaximal inhibitory concentration $\left(\mathrm{IC}_{50}\right)$ value of $0.25 \mu \mathrm{M}$ in BxPC-3 cells and $0.5 \mu \mathrm{M}$ in PANC-1 cells. Notably, NFF cells were markedly less sensitive to JCTH-4 insult (Figure 2C).
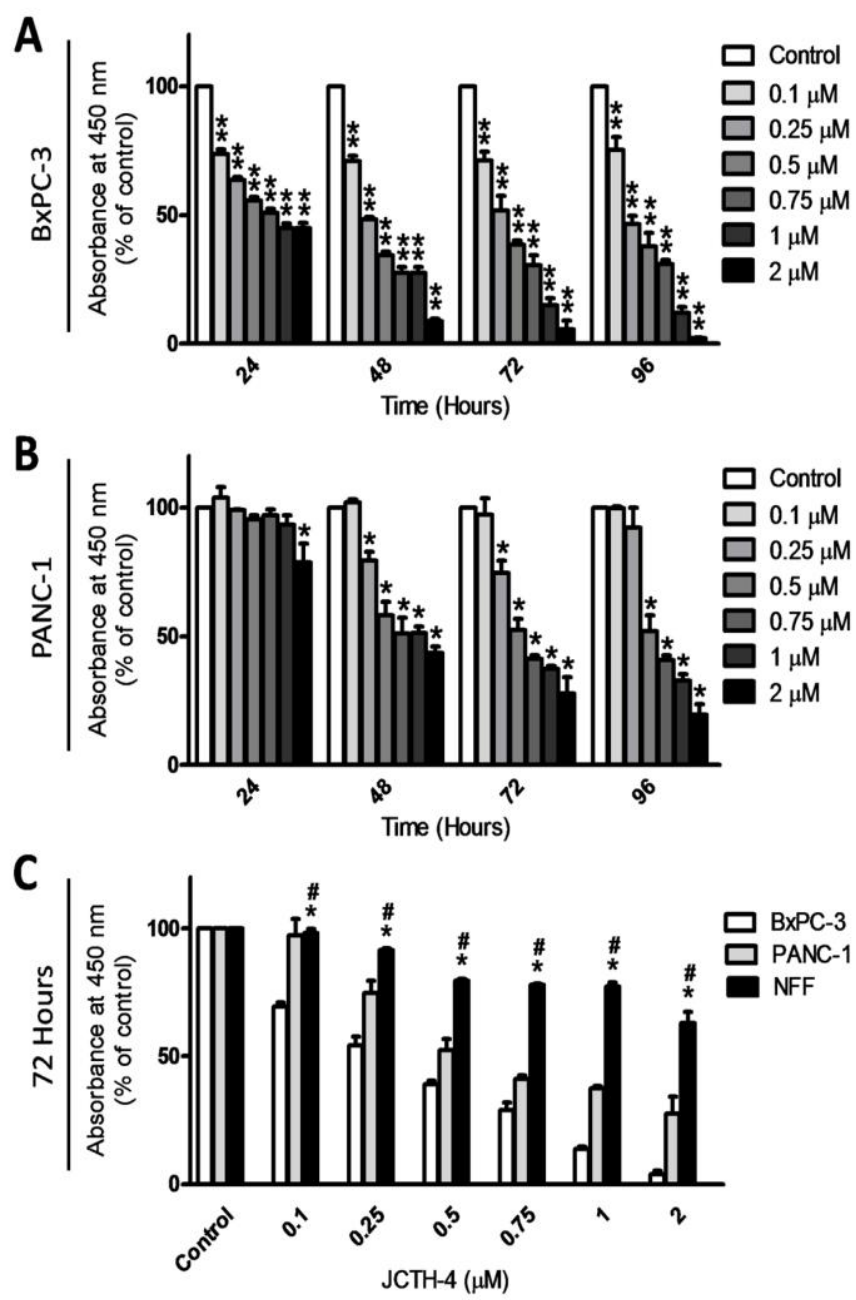

Figure 2: JCTH-4 Selectively Induces Cytotoxicity in Pancreatic Carcinoma Cells in a Time \& DoseDependent Manner. Effect of JCTH-4 on cellular viability of cells was determined by the WST-1 colorimetric assay. (A) BxPC-3 and (B) PANC-1 cells were treated with JCTH-4 at the indicated concentrations and times. The WST-1 reagent was used to quantify cell viability. Absorbance was read at $450 \mathrm{~nm}$ and expressed as a percent of the solvent control $\left(\mathrm{Me}_{2} \mathrm{SO}\right)$. Values are expressed as mean $\pm \mathrm{SD}$ from quadruplicates of 3 independent experiments. $* p<0.05, * * p<0.01$ versus solvent control $\left(\mathrm{Me}_{2} \mathrm{SO}\right)$. (C) Viability of NFF cells treated with JCTH-4 at the indicated concentrations compared to BxPC-3 and PANC-1 cells after 72 hours was determined by the WST-1 colorimetric assay. The WST-1 reagent was used to quantify viability. Absorbance was read at 450 $\mathrm{nm}$ and expressed as a percent of the solvent control $\left(\mathrm{Me}_{2} \mathrm{SO}\right)$. Values are expressed as mean $\pm \mathrm{SD}$ from quadruplicates of 3 independent experiments. ${ }^{*} p<0.01$ versus BxPC- 3 cells, $\# p<0.05$ versus PANC-1 cells. 


\subsection{JCTH-4 Selectively Induces Apoptosis in Pancreatic Carcinoma Cells}

Apoptotic morphology was observed in pancreatic cancer cells treated with $\mathrm{JCTH}-4$ as seen with Hoechst nuclear staining and phase microscopy; cell shrinkage, blebbing, brightly stained and condensed nuclei, and apoptotic bodies were produced by JCTH-4 in BxPC-3 cells at $0.5 \mu \mathrm{M}$ and $1 \mu \mathrm{M}$ and in PANC- 1 cells at $1 \mu \mathrm{M}$ and $2 \mu \mathrm{M}$ (Figure 3A and B). Such morphology was absent in NFF cells treated with JCTH-4 (Figure 3C). Selective induction of apoptosis in pancreatic cancer cells was confirmed with the binding of Annexin $\mathrm{V}$ to exposed phosphatidylserine, a biochemical marker of apoptosis, as indicated by the green fluorescence (Figure 3D-F). These results illustrate JCTH-4 to be a potent and selective agent against BxPC-3 and PANC-1 cells via induction of apoptotic cell death.
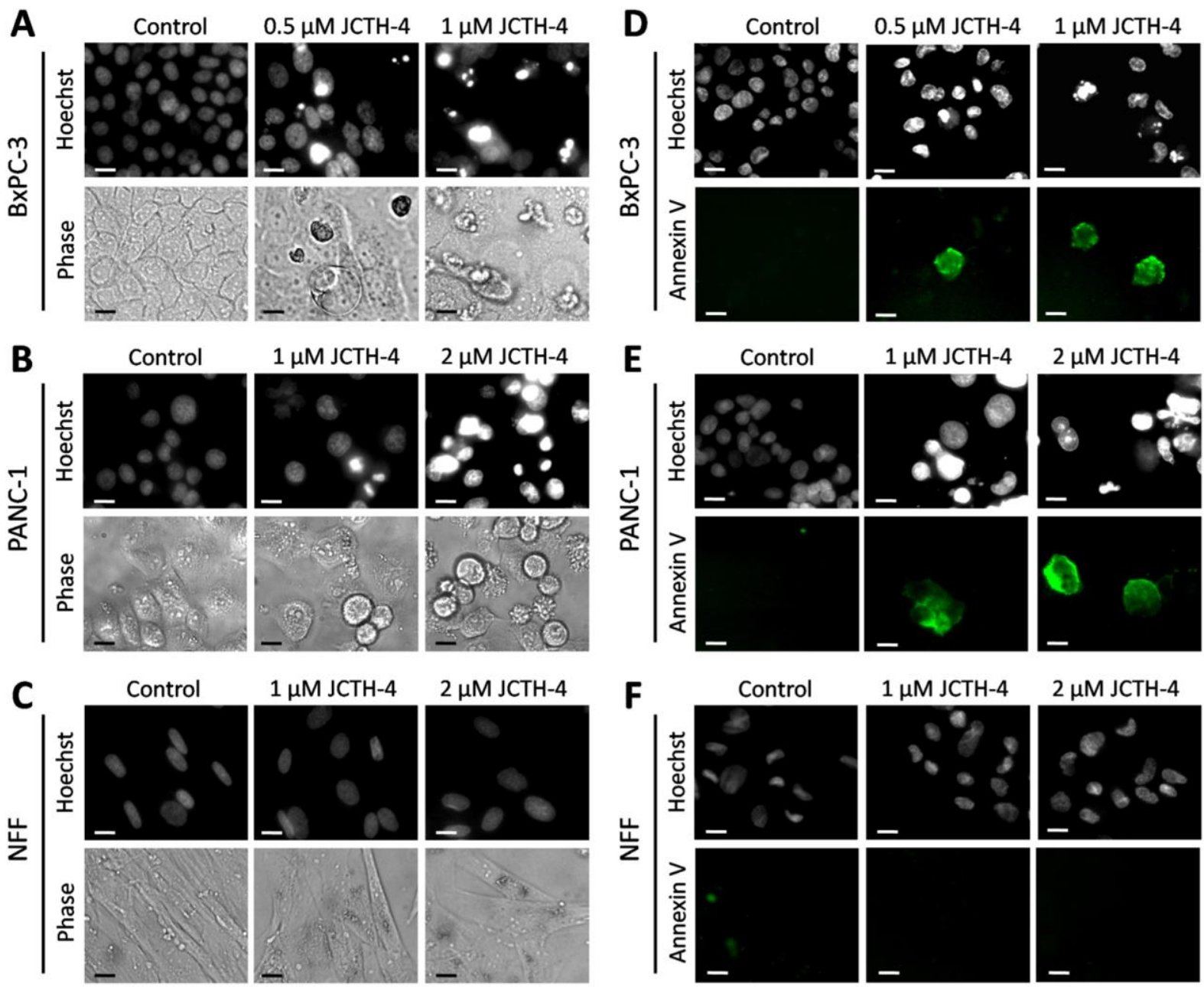

Figure 3: JCTH-4 Selectively Induces Apoptosis in Pancreatic Carcinoma Cells. Nuclear morphology of (A) BxPC-3, (B) PANC-1 and (C) NFF cells after 72 hours of treatment. Cells were treated with JCTH-4 at the indicated concentrations and solvent control $\left(\mathrm{Me}_{2} \mathrm{SO}\right)$. Afterwards, the cells were stained with Hoechst 33342 dye. Images were taken at 400x magnification on a fluorescent microscope. The accompanying phase images are shown below the Hoechst images. Apoptosis is evident in cells with bright and condensed nuclei accompanied by apoptotic bodies. Annexin $\mathrm{V}$ binding to externalized phosphatidylserine, a marker for apoptosis, was monitored in (D) BxPC-3, (E) PANC-1, and (F) NFF cells. Cells were treated with the indicated concentrations of JCTH-4 and solvent control $\left(\mathrm{Me}_{2} \mathrm{SO}\right)$ for 72 hours. Green fluorescence is indicative of Annexin V binding to phosphatidylserine. Cells were also stained with Hoechst 33342 dye. Images were taken at 400x magnification on a fluorescent microscope. Scale bar $=15$ $\mu \mathrm{m}$. 

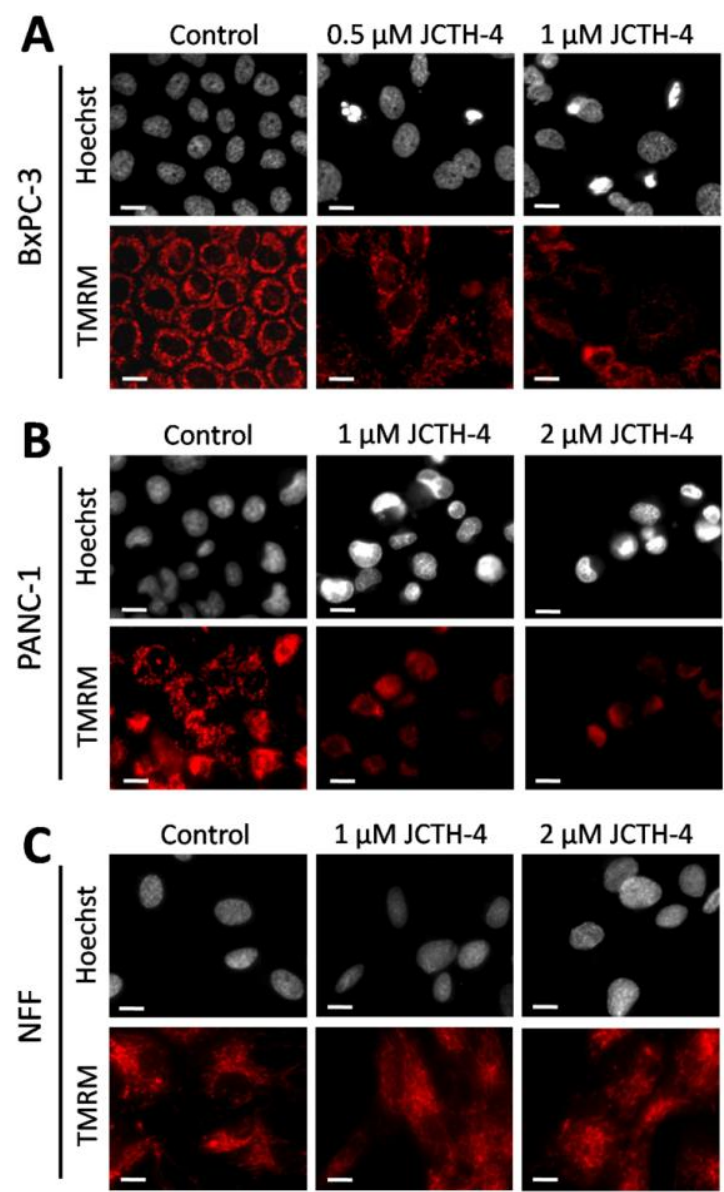

$2 \mu \mathrm{M} \mathrm{JCTH}-4$

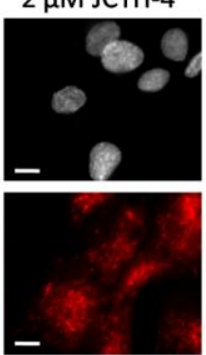

D
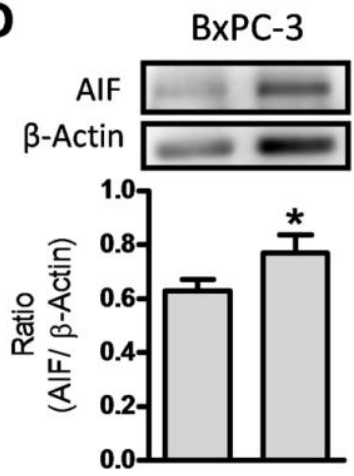

$1 \mu \mathrm{M} \mathrm{JCTH}-4$

E
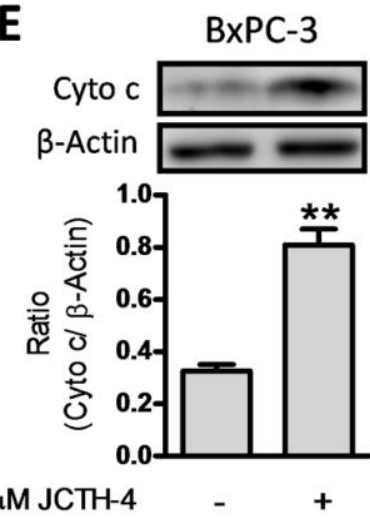

$\mathbf{F}$
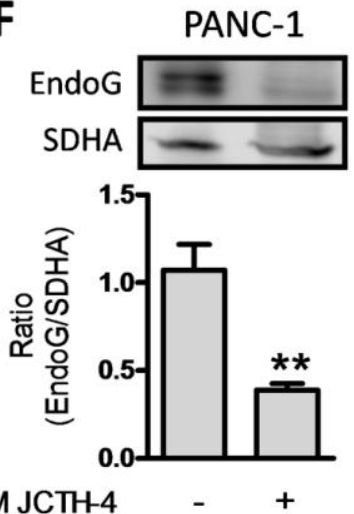

$1 \mu \mathrm{M} \mathrm{JCTH-4}$

$\mathbf{G}$

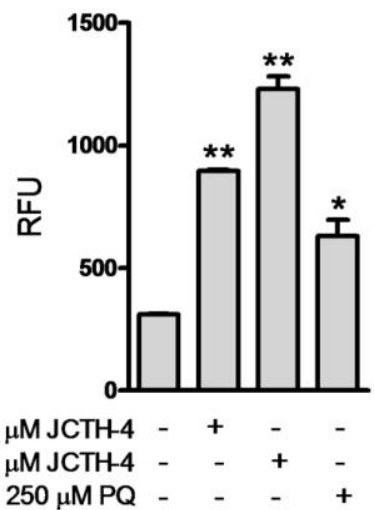

Figure 4: JCTH-4 Selectively Induces Apoptosis by Targeting Mitochondria in Pancreatic Carcinoma Cells. (A) BxPC-3, (B) PANC-1 and (C) NFF cells were treated with the indicated concentrations of JCTH-4 and solvent control $\left(\mathrm{Me}_{2} \mathrm{SO}\right)$ for 72 hours and stained with TMRM and Hoechst dye. Red fluorescent punctuate marks are indicative of mitochondria with intact MMP. Images were taken at 400x magnification on a fluorescent microscope. Scale bar $=15$ $\mu \mathrm{m}$. Cytosolic fractions, separated from mitochondria by centrifugation, of BxPC-3 cells treated with JCTH-4 or solvent control $\left(\mathrm{Me}_{2} \mathrm{SO}\right)$ for 72 hours were monitored for (D) AIF and (E) Cyto c release from mitochondria by western blot analysis; $\beta$-Actin was probed as a loading control. Mitochondrial fractions, produced by centrifugation, of PANC-1 cells treated with JCTH-4 or solvent control $\left(\mathrm{Me}_{2} \mathrm{SO}\right)$ for 72 hours were monitored for the retention of $(\mathrm{F})$ EndoG in the mitochondria by western blot analysis; SDHA was probed as a loading control. Densitometric analyses were performed using ImageJ software and statistics were calculated using GraphPad Prism version 5.0. Values are expressed as mean $\pm \mathrm{SD} .{ }^{*} p<0.05$, $* * p<0.01$ versus solvent control $\left(\mathrm{Me}_{2} \mathrm{SO}\right)$. (G) Isolated mitochondria of $\mathrm{BxPC}-3$ cells were treated directly with JCTH-4 and solvent control $\left(\mathrm{Me}_{2} \mathrm{SO}\right)$. ROS was measured with Amplex Red substrate in the presence of horseradish peroxidase (HRP). Paraquat (PQ) was used as positive control. Fluorescence readings were taken after 2 hours of incubation at Ex. $560 \mathrm{~nm}$ and Em. $590 \mathrm{~nm}$ and were expressed as relative fluorescence units (RFU). Statistics were performed using GraphPad Prism version 5.0. Image is representative of 3 independent experiments demonstrating similar trends. Values are expressed as mean \pm SD of quadruplicates of 1 independent experiment. $* p<0.05, * * p<0.001$ versus solvent control $\left(\mathrm{Me}_{2} \mathrm{SO}\right)$.

\subsection{JCTH-4 Selectively Induces Apoptosis by Targeting Mitochondria in Pancreatic Carcinoma Cells}

In previous reports, we have attributed the apoptosis-inducing capabilities of PST to mitochondrial targeting [17-22]. To substantiate such targeting by JCTH-4, mitochondria in pancreatic cancer cells were monitored following JCTH-4 insult. Following 72 hours of treatment with JCTH-4, cells were evaluated for MMP 
dissipation, an indicator of mitochondrial membrane permeabilization, with TMRM dye. Loss of MMP, illustrated by the decrease of red TMRM fluorescence, was observed in BxPC-3 and PANC-1 cells but not in NFF cells with the indicated concentrations of JCTH-4 (Figure 4AC).

Subsequent to mitochondrial membrane permeabilization, various apoptogenic factors are released from the mitochondria into the cytosol which can then directly or indirectly execute apoptosis [2]. To verify whether or not JCTH-4 is able to cause release of these factors, pancreatic cancer cells were treated with $1 \mu \mathrm{M}$ JCTH-4 for 72 hours. Following treatment, cells were lysed and centrifuged to produce cytosolic and mitochondrial fractions that were analyzed for the release or retention of apoptogenic factors respectively with JCTH-4 insult. Indeed, JCTH-4 caused the release of the apoptogenic factors AIF and Cyto $\mathrm{c}$ from mitochondria of BxPC-3 cells and a decreased retention of the apoptogenic factor EndoG in PANC-1 mitochondria (Figure 4D-F). Complementary to these findings, isolated mitochondria from BxPC-3 cells were treated directly with $1 \mu \mathrm{M}$ JCTH-4 for 2 hours, and exhibited an increase in ROS production, an indicator of mitochondrial dysfunction (Figure 4G). PQ was used as a positive control as it is known to induce ROS generation in mitochondria [24]. Collectively, these findings clearly demonstrate the mitochondria to be the target of JCTH-4.

\subsection{Activity of JCTH-4 is Independent of Caspase Activation}

Upon their expulsion from the mitochondria, apoptogenic factors can execute apoptosis directly or indirectly; factors such as AIF and EndoG are able to translocate to the nucleus to execute apoptosis directly while Cyto c activates a caspase signalling cascade to achieve apoptosis $[2,25,26,3]$. To assess the involvement of caspases in JCTH-4-induced apoptosis, the broad spectrum caspase inhibitor Z-VAD-FMK was used in conjunction with $1 \mu \mathrm{M}$ JCTH- 4 on BxPC-3 cells for 72 hours. As seen using the WST-1 colorimetric assay for cell viability, the inhibitor was unable to protect the BxPC-3 cells from
JCTH-4, demonstrating a caspase-independent mode of action (Figure 5).

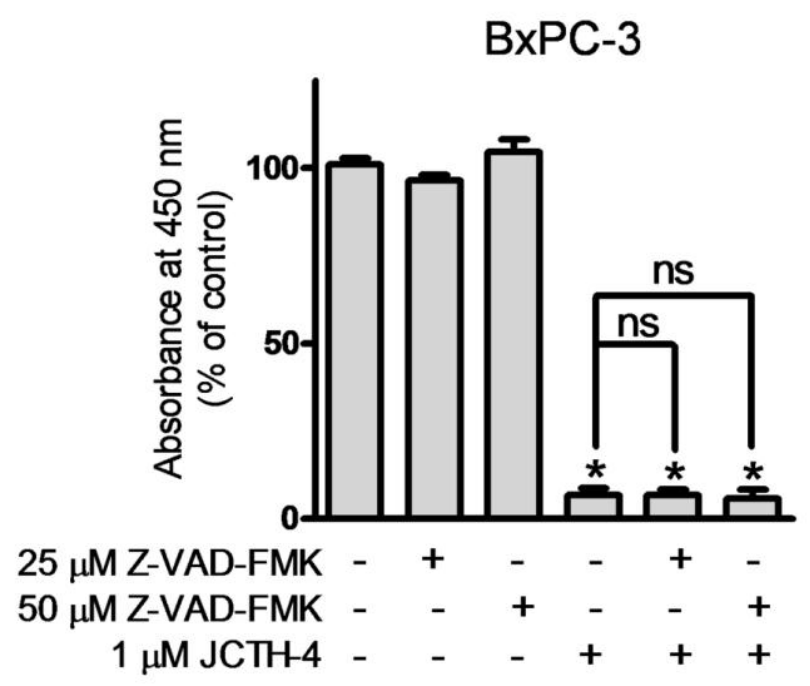

Figure 5: Activity of JCTH-4 is Independent of Caspase Activation. Effect of broad spectrum caspase inhibition on JCTH-4-induced cytotoxicity in pancreatic cancer cells. BxPC-3 cells were treated with Z-VAD-FMK, a broad spectrum caspase inhibitor, at the indicated concentrations with and without the presence of $1 \mu \mathrm{M} \mathrm{JCTH}-4$ treatment for 72 hours and the WST-1 reagent was used to quantify cell viability. Absorbance was read at $450 \mathrm{~nm}$ and expressed as a percent of solvent control $\left(\mathrm{Me}_{2} \mathrm{SO}\right)$. Values are expressed as mean \pm SD from quadruplicates of 3 independent experiments. ${ }^{*} p<0.001$ versus solvent control $\left(\mathrm{Me}_{2} \mathrm{SO}\right)$; $\mathrm{ns}=$ not significant.

\subsection{Selective Autophagic Induction Occurs in Pancreatic Carcinoma Cells Treated with JCTH-4}

Both pro-death and pro-survival autophagic responses have been implicated with chemotherapeutic insult [4]. As JCTH-4 causes oxidative stress in pancreatic cancer cells, a known inducer of autophagy, we monitored pancreatic cancer cells for autophagic induction. PANC-1 cells were treated with TAM, a known inducer of autophagy as a positive control, and JCTH- 4 at 1 and $2 \mu \mathrm{M}$ for 72 hours and stained with MDC, an indicator of autophagosomes. Bright blue punctate MDC staining was present in cells treated with JCTH-4, comparable to the TAM treated cells (Figure 6A). PANC-1 cells were also stained with PI, but none of the aforementioned treatments yielded positive PI 
staining (Figure 6A). Interestingly, JCTH-4 did not induce autophagy in NFF cells as seen with MDC staining (Figure 6B).

The conversion of microtubule-associated protein1 light chain 3 (LC3) localized in the cytosol as LC3-I, to LC3-II which is recruited to autophagosomal membranes occurs with the induction of autophagy [27]. To validate autophagic induction by JCTH-4 in PANC-1 cells, western blot analyses were performed on cell lysates of PANC-1 cells treated with $1 \mu \mathrm{M} \mathrm{JCTH-}$ 4 for 72 hours to analyze the conversion of LC3-I to LC3-II; this conversion was observed with JCTH-4 treatment and produced at greater autophagic response than the TAM treated cells used as a positive control (Figure 6C). Thus, JCTH-4 is capable of selectively inducing apoptosis in pancreatic cancer cells.
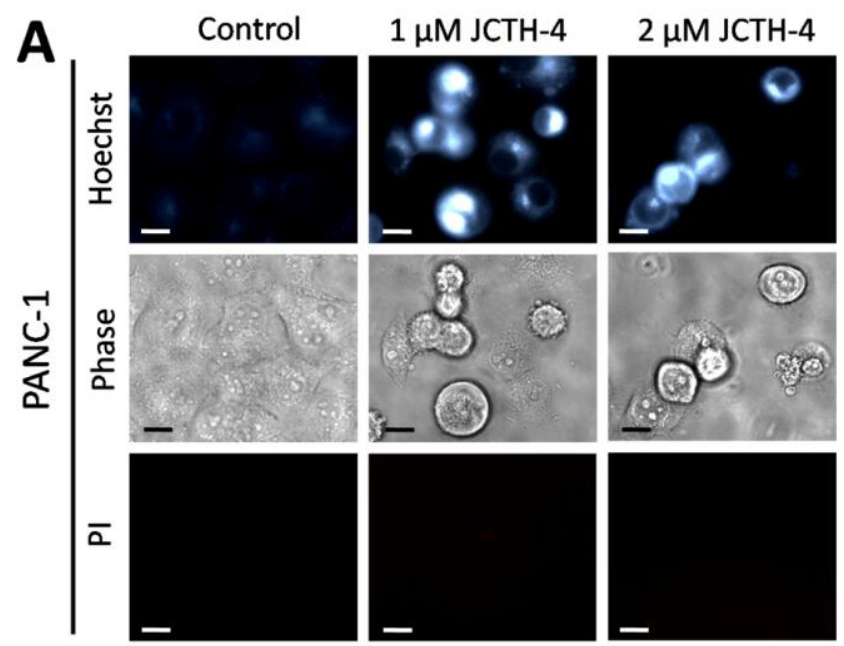

B
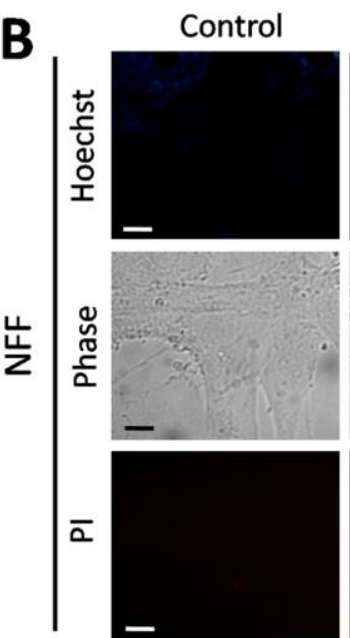

$0.5 \mu \mathrm{M}$ JCTH-4
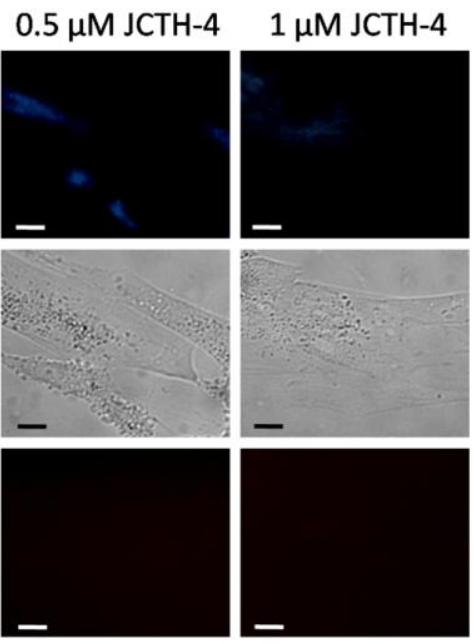
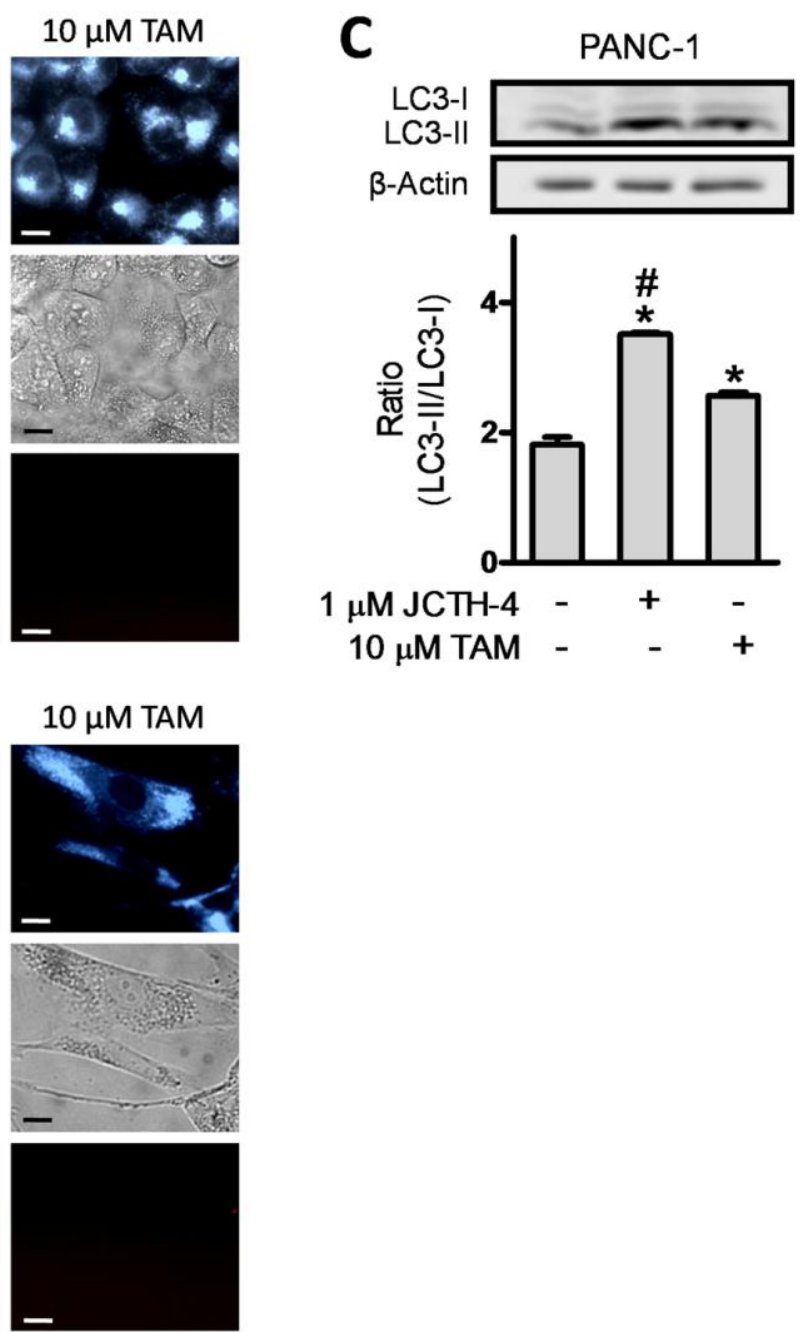

Figure 6: Selective Autophagic Induction Occurs in Pancreatic Carcinoma Cells Treated with JCTH-4. (A) PANC-1 and (B) NFF cells were stained with MDC after treatment with JCTH-4, TAM, or solvent control $\left(\mathrm{Me}_{2} \mathrm{SO}\right)$ at the indicated concentrations for 72 hours. Bright blue MDC punctate marks are indicative of autophagic vacuoles. Accompanying phase and propidium iodide-stained images are shown below the MDC images. Scale bar $=15 \mu \mathrm{m} .(\mathrm{C})$ Western Blot analysis were performed on lysates of PANC-1 cells treated with JCTH-4, TAM, and solvent control $\left(\mathrm{Me}_{2} \mathrm{SO}\right)$ at the indicated concentrations for 72 hours to monitor the conversion of LC3-I to LC3-II, a marker of autophagy. $\beta$-actin was probed as a loading control. Densitometric analyses were done using ImageJ software and statistics were calculated using GraphPad Prism version 5.0. Values are expressed as mean \pm SD. $* p<0.01$ versus solvent control $\left(\mathrm{Me}_{2} \mathrm{SO}\right)$; $\# p<0.01$ versus $10 \mu \mathrm{M}$ TAM. 


\section{Discussion}

Currently, pancreatic cancer stands as one of the most fatal malignancies in the world $[9,10]$. As this disease lacks distinct and evident symptoms, it is difficult to diagnose in its early stages. Thus, pancreatic cancer is often found in its advanced stages with most patients showing metastases to the liver, lungs, or proximal lymph nodes at the time of diagnosis [11,12]. Very few anti-cancer drugs are available for the treatment of this disease as most standard chemotherapeutics have been shown to be ineffective; doxorubicin, 5-5fluorouracil, and cisplatin have been shown to be ineffective both in vitro and in vivo [28]. The nucleoside analogue gemcitabine has been shown to be moderately effective and currently serves as the standard chemotherapeutic treatment for pancreatic cancer [29]. Despite various efforts to enhance the effectiveness of this drug with other chemotherapeutics such as erlotinib, gemcitabine still remains only moderately potent against pancreatic cancer and is often rendered ineffective with the onset of chemoresistance [30,29]. Consequently, the necessity for a more potent treatment presently remains unfulfilled.

In this study, we report the activity of a synthetic analogue of PST, JCTH-4, against human pancreatic cancer cells. JCTH-4 was able to effectively induce cytotoxicity in the BxPC-3 and PANC-1 pancreatic cancer cells by way of apoptotic induction in a selective manner. Necrotic cell death, characterized by lysis of the plasma membrane, by JCTH-4 was ruled out as cells subjected to JCTH-4 were negative for PI staining, a DNA stain impermeable to the plasma membrane (Figure 6A) [31]. The genotoxic drug gemcitabine is the only chemotherapeutic that has shown moderate effectiveness against pancreatic cancer. Giroux et al., 2006 demonstrated that gemcitabine is effective in BxPC-3 cells at submicromolar concentrations; however, the effectiveness of this drug quickly plateaus as the dose is increased [29]. Furthermore, they observed that PANC-1 cells have minimal sensitivity to gemcitabine with an $\mathrm{IC}_{50}$ value of $25 \mu \mathrm{M}$ after 72 hours [29]. In contrast, the $\mathrm{IC}_{50}$ values for BxPC-3 and PANC-1 cells were $0.25 \mu \mathrm{M}$ and $0.5 \mu \mathrm{M}$ respectively after 72 hours (Figure $2 \mathrm{~A} \& 2 \mathrm{~B}$ ).
Thus, JCTH-4 is more effective against both BxPC-3 and PANC-1 cells than gemcitabine. Similar to PST, JCTH-4 is selective against cancer cells as we have previously shown minimal toxicity in normal human fibroblasts and noncancerous peripheral blood mononuclear cells [23]. Additionally, in this study NFF cells proved to be markedly less sensitive to JCTH-4 compared to both BxPC-3 and PANC-1 cells (Figure 2C), did not exhibit any observable signs of apoptotic induction (Figure 3F), and were not affected in terms of their dividing capacity after removal of JCTH-4 (Data not shown). On the contrary, other chemotherapeutics currently available such as Taxol and VP-16 have been shown to produce cytotoxicity in normal human fibroblasts at their effective doses [17]. Thus, these findings illustrate JCTH-4 to be a potentially more effective chemotherapeutic alternative to current cancer therapies.

Previously, we have shown the natural compound PST to be a potent and selective anticancer agent; PST was able to induce apoptosis in numerous cancer cell types with minimal cytotoxicity in non-cancerous cells [16-20]. Furthermore, PST was able to decrease the volume of human tumor xenografts in vivo and was nontoxic to mice [21,22]. PST however, is only present in parts per million quantities in the buds of the Hymenocallis littoralis plant and many difficulties have burdened its chemical synthesis. In an attempt to circumvent these issues, we subsequently synthesized various analogues of PST and found similar anti-cancer activity with JCTH-4 in human neuroblastoma and leukemia cell lines, with no evident toxicity to various noncancerous cell types [23]. The findings presented in this study demonstrate JCTH-4 to also be a potent agent against BxPC-3 and PANC-1 cells, two different cell lines of a very aggressive and deadly cancer. Thus, previous limitations hindering the preclinical and clinical work with PST have been surpassed.

As with PST, our results outline the mechanistic target of JCTH-4 to be the mitochondria; JCTH-4 was shown to dissipate MMP (Figure 4A \& B), cause the release of apoptogenic factors from the mitochondria (Figure 4D \& E), and in isolated mitochondria, increase 
ROS production (Figure 4G). Such mitochondrial targeting was not evident in NFF cells (Figure 4C), complementing previous work with PST; mitochondrial ROS production selectively increased in isolated mitochondria of cancer cells but not in normal human fibroblast mitochondria with PST treatment [17]. In response to various forms of cellular stress, the tumor suppressor p53 transcriptionally activates various proapoptotic proteins that can give rise to mitochondrial membrane permeabilization [32]. Although moderately differing in sensitivities, both BxPC-3 and PANC-1, two different p53-mutated pancreatic cancer cell lines, were responsive to JCTH-4 [33,34]. Thus, signalling of p53 and its transcriptional targets, all of which are cellular events upstream of mitochondrial permeabilization, play an insignificant role in JCTH-4-induced apoptosis, supporting the notion of a direct mitochondrial target by JCTH-4. The caspase-independent nature of JCTH-4-induced apoptosis (Figure 5) further supports mitochondrial targeting by JCTH-4 as caspase signalling can occur upstream of the mitochondria to permeabilize it. Although caspases are required for the execution apoptosis through Cyto c, JCTH4 may yield apoptosis through the release of other apoptogenic factors such as AIF and EndoG which can directly execute apoptosis in a caspaseindependent manner [2,25,26,3].

Various differences exist between normal and cancer cell mitochondria which may serve as the basis by which JCTH-4 targets cancer cells specifically. The Warburg effect, a phenomenon in which cancer cells rely heavily on glycolysis for ATP generation, is postulated to be a result of mitochondrial dysfunction [35]. It is therefore possible for JCTH-4 to target these differences in metabolism. Moreover, an acidic cytosolic environment is created as result these alterations in metabolic activity which in part contributes to elevated MMP in cancer cell mitochondria. Heightened MMP in cancer cell mitochondria has been linked to an increased capacity to evade apoptosis, invade neighbouring tissue, and promote angiogenesis [36]. It is this elevation in MMP however, that may be exploited by JCTH-4 for cancer selectivity. Upon entering the cell, JCTH-4 may acquire as positive charge through enzymatic processing which may then be preferentially taken up selectively by cancer cell mitochondria as a result of their increased MMP. Additionally, cancer cells have been reported to overexpress various proteins which act to inhibit mitochondrial outer membrane permeabilization such as antiapoptotic proteins of the Bcl-2 family, peripheral-type benzodiazepine, and hexokinase II [37-39]. All of these proteins could serve as potential targets of JCTH-4.

Cellular stress, such as oxidative stress, can trigger the induction of autophagy. As JCTH-4 was found to increase the production of ROS in isolated mitochondria of pancreatic cancer cells (Figure 4G), we monitored PANC-1 for autophagic induction following JCTH-4 insult. Indeed, autophagy was induced selectively in pancreatic cancer cells by JCTH-4 (Figure 6A-C). Increased ROS production triggered by JCTH-4induced mitochondrial dysfunction is most likely responsible for selective autophagic induction in these pancreatic cancer cells, which provides additional evidence of a mitochondrial target by JCTH-4. Nevertheless, these cells ultimately die as a result of mitochondrial membrane permeabilization and the subsequent release of apoptogenic factors.

In summary, the novel analogue of PST, JCTH-4, is effective in inducing apoptosis and autophagy selectively in pancreatic cancer cells by means of mitochondrial targeting. Furthermore, this compound was shown to be very effective against one of the most aggressive and notoriously chemoresistant malignancies, surpassing its current standard chemotherapeutic agent in efficacy. Thus, the unprecedented activity of this compound presents a potentially safe and effective treatment for pancreatic cancer.

\section{Acknowledgements}

This work has been supported by a CIHR Frederick Banting and Charles Best Canada Graduate Scholarship awarded to Dennis Ma and donations from the Knights of Columbus Chapter 9671 (Windsor, Ontario), and Dave and Donna Couvillon in memory of Kevin Couvillon. Thank you to Ian Tuffley for the critical review of this manuscript. 


\section{References}

1. Hengartner MO. The biochemistry of apoptosis. Nature 2000; 407(6805):770-6. DOI: $10.1038 / 35037710$

2. Earnshaw WC. Apoptosis. A cellular poison cupboard. Nature 1999; 397(6718):387- 389. DOI: $10.1038 / 17015$

3. Degterev A, Boyce M, Yuan J. A decade of caspases. Oncogene 2003; 22(53):8543-67. DOI:10.1038/sj.onc.1207107

4. Dalby KN, Tekedereli I, Lopez-Berestein G, Ozpolat B. Targeting the prodeath and prosurvival functions of autophagy as novel therapeutic strategies in cancer. Autophagy 2010; 6(3): 322-9. [Epub 2010 Apr 26] DOI: org/10.4161/auto.6.3.11625

5. Kroemer G, Mariño G, Levine B. Autophagy and the integrated stress response. Mol Cell. 2010; 40(2):280-93. 10.1016/j.molcel.2010.09.023

6. Xie Z, Klionsky DJ. Autophagosome formation: core machinery and adaptations. Nat Cell Biol. 2007; 9(10):1102-9. DOI: 10.1038/ncb10071102

7. Borst P, Rottenberg S. Cancer cell death by programmed necrosis? Drug Resist Updat. 2004; 7(6):321-4. [Epub 2005 Jan 11] DOI: 10.1016/j.drup.2004.11.003

8. Liu JJ, Lin M, Yu JY, Liu B, Bao JK. Targeting apoptotic and autophagic pathways for cancer therapeutics. Cancer Lett. 2011; 300(2):10514. [Epub 2010 Oct 30] DOI: 10.1016/j.canlet.2010.10.001

9. Eckel F, Schneider G, Schmid RM. Pancreatic cancer: a review of recent advances. Expert Opin Investig Drugs 2006; 15:1395-1410. DOI: $10.1517 / 13543784.15 .11 .1395$

10. Matsuno S, Egawa S, Fukuyama S, Motoi F, Sunamura M, Isaji S, Imaizumi T, Okada S, Kato H, Suda K, Nakao A, Hiraoka T, Hosotani R, Takeda K. Pancreatic cancer registry in Japan: 20 years of experience. Pancreas 2004; 28:219-230.

11. Hruban RH. Pancreatic cancer: from genes to patient care. J. Gastrointest. Surg. 2001;
$5: 583-587$. DOI:10.1016/S1091-

255X(01)80099-8

12. Jemal A, Siegel R, Xu J, Ward E. Cancer statistics, 2010. CA Cancer J. Clin. 2010; 60:277-300. DOI: 10.3322/caac.20073

13. Burris HA 3rd, Moore MJ, Andersen J, Green MR, Rothenberg ML, Modiano MR, Cripps MC, Portenoy RK, Storniolo AM, Tarassoff P, Nelson R, Dorr FA, Stephens CD, Von Hoff DD Improvements in survival and clinical benefit with gemcitabine as first-line therapy for patients with advanced pancreas cancer: a randomized trial. J Clin Oncol 1997; 15:24032413.

14. Sultana A, Ghaneh P, Cunningham D, Starling N, Neoptolemos JP, Smith CT. Gemcitabine based combination chemotherapy in advanced pancreatic cancer-indirect comparison. BMC Cancer 2008; 8:192. DOI: 10.1186/1471-24078-192

15. Cunningham D, Chau I, Stocken DD, Valle JW, Smith D, Steward W, Harper PG, Dunn J, Tudur-Smith C, West J, Falk S, Crellin A, Adab F, Thompson J, Leonard P, Ostrowski J, Eatock M, Scheithauer W, Herrmann R, Neoptolemos JP. Phase iii randomized comparison of gemcitabine versus gemcitabine plus capecitabine in patients with advanced pancreatic cancer. J Clin Oncol 2009; 27(33):5513-5518. 10.1200/JCO.2009.24.2446

16. Kekre N, Griffin C, McNulty J, Pandey S. Pancratistatin causes early activation of caspase- 3 and the flipping of phosphatidyl serine followed by rapid apoptosis specifically in human lymphoma cells. Cancer Chemother Pharmacol 2005; 56(1): 29-38. DOI: 10.1007/s00280-004-0941-8

17. McLachlan A, Kekre N, McNulty J, Pandey S. Pancratistatin: a natural anti-cancer compound that targets mitochondria specifically in cancer cells to induce apoptosis. Apoptosis 2005; 10(3):619-630. DOI: 10.1007/s10495-0051896-x

18. Siedlakowski P, McLachlan-Burgess A, Griffin C, Tirumalai SS, McNulty J, Pandey S. Synergy of Pancratistatin and Tamoxifen on breast cancer cells in inducing apoptosis by targeting mitochondria. Cancer Biol Ther 
2007;

$7(3): 376-384$.

DOI:

org/10.4161/cbt.7.3.5364

19. Chatterjee SJ, McNulty J, Pandey S. Sensitization of human melanoma cells by tamoxifen to apoptosis induction by pancratistatin, a nongenotoxic natural compound. Melanoma Res. 2010 [Epub 2010 Mar 17 ahead of print]

20. Griffin C, Hamm C, McNulty J, Pandey S. Pancratistatin induces apoptosis in clinical leukemia samples with minimal effect on noncancerous peripheral blood mononuclear cells. Cancer Cell Int. 2010; 10:6. DOI: 10.1186/1475-2867-10-6

21. Griffin C, Karnik A, McNulty J, Pandey S. Pancratistatin selectively targets cancer cell mitochondria and reduces growth of human colon tumor xenografts. Molecular Cancer Therapeutics 2011a; 10(1):57-68. DOI: 10.1158/1535-7163.MCT-10-0735

22. Griffin C, McNulty J, Pandey S. Pancratistatin induces apoptosis and autophagy in metastatic prostate cancer cells. Int J Oncol. 2011b; 38(6):1549-56. DOI: 10.3892/ijo.2011.977

23. Collins J, Rinner U, Moser M, Hudlicky T, Ghiviriga I, Romero AE, Kornienko A, Ma D, Griffin C, Pandey S. Chemoenzymatic synthesis of Amaryllidaceae constituents and biological evaluation of their C-1 analogues. The next generation synthesis of 7deoxypancratistatin and transdihydrolycoricidine. J Org Chem 2010; 75(9):3069-84. DOI: 10.1021/jo1003136

24. Cochemé HM, Murphy MP. Complex I is the major site of mitochondrial superoxide production by paraquat. J Biol Chem. 2008; 283(4):1786-98. [Epub 2007 Nov 26] DOI: $\underline{10.1074 / \mathrm{jbc} . M 708597200}$

25. Susin SA, Lorenzo HK, Zamzami N, Marzo I, Snow BE, Brothers GM, Mangion J, Jacotot E, Costantini P, Loeffler M, Larochette N, Goodlett DR, Aebersold R, Siderovski DP, Penninger JM, Kroemer G. Molecular characterization of mitochondrial apoptosisinducing factor. Nature 1999; 397(6718):4416. DOI: $10.1038 / 17135$

26. Li LY, Luo X, Wang X. Endonuclease G is an apoptotic DNase when released from mitochondria. Nature 2001; 12(6842):95-9. DOI: $10.1038 / 35083620$

27. Kabeya Y, Mizushima N, Ueno T, Yamamoto A, Kirisako T, Noda T, Kominami E, Ohsumi Y, Yoshimori T. LC3, a mammalian homologue of yeast Apg8p, is localized in autophagosome membranes after processing. EMBO J. 2000; 19(21):5720-8. DOI: $\underline{10.1093 / \mathrm{emboj} / 19.21 .5720}$

28. Schultz RM, Merriman RL, Toth JE, Zimmermann JE, Hertel LW, Andis SL, Dudley DE, Rutherford PG, Tanzer LR, Grindey GB. Evaluation of new anticancer agents against the MIA PaCa-2 and PANC-1 human pancreatic carcinoma xenografts. Oncol Res. 1993; 5(6-7):223-8.

29. Giroux V, Malicet C, Barthet M, Gironella M, Archange C, Dagorn JC, Vasseur S, Iovanna JL. p8 is a new target of gemcitabine in pancreatic cancer cells. Clin Cancer Res. 2006; 12(1):235-41. DOi: 10.1158/1078-0432.CCR$\underline{05-1700}$

30. Squadroni M, Fazio N. Chemotherapy in pancreatic adenocarcinoma. Eur Rev Med Pharmacol Sci. 2010; 14(4):386-94.

31. Pollack M, Leeuwenburgh C. Apoptosis and Aging: Role of the Mitochondrial. Journal of Gerontology 2001; 11:475-482.

32. Zilfou JT, Lowe SW. Tumor suppressive functions of p53. Cold Spring Harb Perspect Biol. 2009; 1(5):a001883. DOI: 10.1101/cshperspect.a001883

33. Berrozpe G, Schaeffer J, Peinado MA, Real FX, Perucho M. Comparative analysis of mutations in the p53 and K-ras genes in pancreatic cancer. Int $\mathbf{J}$ Cancer 1994; 58(2):185-91.

34. Butz J, Wickstrom E, Edwards J. Characterization of mutations and loss of heterozygosity of p53 and K-ras2 in pancreatic cancer cell lines by immobilized polymerase chain reaction. BMC Biotechnol. 2003; 3:11. [Epub 2003 Jul 23]. DOI: 10.1186/1472-6750$\underline{3-11}$

35. Warburg O. On the origin of cancer cells. Science 1956; 123(3191):309-14. DOI:10.1126/science.123.3191.309

36. Heerdt BG, Houston MA, Augenlicht LH. Growth properties of colonic tumor cells are a 
function of the intrinsic mitochondrial membrane potential. Cancer Res. 2006; 66(3):1591-6. DOI: 10.1158/0008-5472.CAN05-2717

37. Green DR, Kroemer G. The pathophysiology of mitochondrial cell death. Science 2004; 305(5684):626-9.

DOI: 10.1126/science. 1099320

38. Casellas P, Galiegue S, Basile AS. Peripheral benzodiazepine receptors and mitochondrial function. Neurochem Int. 2002; 40(6):475-86. DOI: $10.1016 / S 0197-0186(01) 00118-8$
39. Mathupala SP, Rempel A, Pedersen PL. Aberrant glycolytic metabolism of cancer cells: a remarkable coordination of genetic, transcriptional, post-translational, and mutational events that lead to a critical role for type II hexokinase. J Bioenerg Biomembr. 1997; 29(4):339-43. 